\title{
New Standards for Clinical Decision Support: A Survey of The State of Implementation
}

\author{
Peter Taber', Christina Radloff', Guilherme Del Fiol', Catherine Staes ${ }^{1,2}$, Kensaku Kawamoto' \\ 1 Department of Biomedical Informatics, University of Utah, Salt Lake City, UT, USA \\ 2 College of Nursing, University of Utah, Salt Lake City, UT, USA
}

\begin{abstract}
Summary
Objectives: To review the current state of research on designing and implementing clinical decision support (CDS) using four current interoperability standards: Fast Healthcare Interoperability Resources (FHIR); Substitutable Medical Applications and Reusable Technologies (SMART); Clinical Quality Language (CQL); and CDS Hooks.

Methods: We conducted a review of original studies describing development of specific CDS tools or infrastructures using one of the four targeted standards, regardless of implementation stage. Citations published any time before the literature search was executed on October 21, 2020 were retrieved from PubMed. Two reviewers independently screened articles and abstracted data according to a protocol designed by team consensus.

Results: Of 290 articles identified via PubMed search, 44 were included in this study. More than three quarters were published since 2018. Forty-three (98\%) used FHIR; 22 (50\%) used SMART; two (5\%) used CQL; and eight (18\%) used CDS Hooks. Twenty-four (55\%) were in the design stage, 15 (34\%) in the piloting stage, and five $(11 \%)$ were deployed in a real-world setting. Only 12 (27\%) of the articles reported an evaluation of the technology under development. Three of the four articles describing a deployed technology reported an evaluation. Only two evaluations with randomized study components were identified. Conclusion: The diversity of topics and approaches identified in the literature highlights the utility of these standards. The infrequency of reported evaluations, as well as the high number of studies in the design or piloting stage, indicate that these technologies are still early in their life cycles. Informaticists will require a stronger evidence base to understand the implications of using these standards in CDS design and implementation.
\end{abstract}

\section{Keywords}

Health information interoperability, clinical decision support, FHIR, SMART, CQL, CDS hooks

Yearb Med Inform 2021:159-71

http://dx.doi.org/10.1055/s-0041-1726502

\section{Introduction}

The management of information in clinical settings has long been recognized by designers of clinical decision support (CDS) tools to be a highly complex problem $[1,2]$. For roughly the last decade, the HITECH Act has led to rapid adoption of electronic health record (EHR) systems [3]. While the HITECH Act envisioned interoperability of data across EHR systems, there have continued to be challenges with the interoperable exchange of clinical data extracted from the proprietary data models of different EHR systems. Efforts by standards organizations such as Health Level Seven International (HL7) have sought to address this issue by offering EHR-agnostic services, data models and languages to improve interoperability across EHRs and organizations [4-6].

In this literature survey, we examine the state of research on the implementations of four current HL7 standards for healthcare information in the context of CDS: Fast Healthcare Interoperability Resources (FHIR); Substitutable Medical Applications and Reusable Technologies (SMART); Clinical Quality Language (CQL); and CDS Hooks. We chose SMART and FHIR because both are now federally regulated standards in the United States. Additionally, CQL is now the standard used by the U.S. Centers for Medicare \& Medicaid Services (CMS) for specifying electronic clinical quality measures. We further added CDS Hooks so that our review could examine a full suite of integrated standards that allow CDS sharing at multiple levels (e.g., logic, apps, services). Previous CDS standards were developed in isolation and were not always interopera- ble among themselves. Beyond these core issues, we opted to examine HL7's standards because it is the largest international health information technology standards development organization worldwide and has potential relevance anywhere EHRs are being implemented. Before describing our review process, we briefly summarize each of these four standards. Further detailed background on these HL7 standards can be found in Strasberg et al. [7].

\subsection{Fast Healthcare Interoperability Resources}

Fast Healthcare Interoperability Resources (FHIR) is a standard that allows different systems to exchange healthcare data in a standard format. The core of the standard consists of "resources", which define commonly used healthcare concepts and relationships. FHIR is distinguished from previous HL7 standards by its use of a Representation State Transfer (REST) application programming interface (API), and the ability to share resources in Extensible Markup Language (XML), JavaScript Object Notation (JSON) or Resource Description Framework (RDF). To avoid over-specification of the standard, resources desired by $80 \%$ or more of developers are intended to be included as a part of the core specification, whereas those less commonly used are expected to require additional specification, for example in what are known as FHIR profiles. The first draft of FHIR was published in 2014. The most recent version as of December 2020, Version 4.0.1, was published in October $2019[5,6,8,9]$. 


\subsection{Substitutable Medical Applications and Reusable Technologies}

Substitutable Medical Applications and Reusable Technologies (SMART) is a standard designed to enable the integration of EHR-agnostic medical applications within EHR systems using existing Web standards. Since its development starting in 2010, SMART has often been used in conjunction with FHIR in a configuration known as "SMART on FHIR" $[9,10]$. SMART enables EHR users to launch add-on apps within the EHR through a single sign-on mechanism, while FHIR enables these apps to exchange patient data with the EHR in a standard data format.

\subsection{Clinical Quality Language}

Clinical Quality Language (CQL) is a human-readable expression language first published as a draft HL7 standard in 2015 that is intended for the representation of CDS logic and quality measures. The language is organized in terms of artifacts that include three components: metadata, clinical quality information and expression logic. CQL provides a standard approach to representing the "if" component of "if-then" decision rules. CQL also can be used to specify a data model and the specific patient data needed $[11,13]$. While not required, CQL implementations often use FHIR as the data model.

\subsection{Clinical Decision Support Hooks}

Clinical Decision Support (CDS) Hooks is a specification first balloted in HL7 in 2018 for provisioning CDS within the EHR via Web services. "Hooks" are events that are automatically triggered upon certain user actions within the EHR, such as opening a patient's chart or ordering a medication. A CDS Hooks request embeds information about the context of clinical workflow, a set of data requested by the CDS service ("prefetch" mechanism), and access to the EHR's FHIR server for additional data requests as needed. CDS services return "cards" to the EHR that may include relevant information (e.g., a patient assessment), actionable suggestions (e.g., a medication order), or links to SMART on FHIR apps relevant to the current clinical process. CDS Hooks uses FHIR to meet its data needs $[14,15]$.

\section{Objectives}

To inform ongoing efforts to implement interoperable CDS within clinical environments by surveying the current state of research on designing and implementing CDS using the HL7 FHIR, SMART, CQL and CDS Hooks standards, including types of CDS used, domains of application and stage of development of current interventions.

\section{Methods}

\subsection{Inclusion Criteria}

The review included research on the use of FHIR, SMART, CQL, or CDS Hooks for CDS. We followed Osheroff et al. [1] in defining CDS as "a process for enhancing health-related decisions and actions with pertinent, organized clinical knowledge and patient information to improve health and healthcare delivery". The review included studies both on infrastructure development (e.g., work intended to support a wide range of different CDS interventions), and on the development of problem-specific CDS tools regardless of development stage. Healthcare professional- and patient-facing tools were both included. Any professionals such as physicians, advanced practice clinicians, pharmacists or nurses were considered healthcare professionals for the purposes of this study.

\subsection{Exclusion Criteria}

We excluded viewpoint articles; reviews; research-focused applications without a significant focus on CDS; public health applications without a significant focus on CDS; non-English language articles; and articles that lacked sufficient detail to populate the majority of our data extraction fields.
Finally, we excluded: i) articles reporting on application or data clearinghouses that did not emphasize CDS applications; ii) general standards development or interoperability efforts not explicitly focused on CDS; and iii) tools focused solely on patient data collection without a clear CDS component.

\subsection{Literature Search}

Medical Subject Heading (MeSH) synonyms for CDS yielded poor results in terms of their relevance and failed to capture articles related to CDS Hooks and CQL. For these reasons, we opted to use the names of the standards instead of MeSH terms. The PubMed database was searched in October 2020 using the following search strategy with no date limits:

("Substitutable Medical Applications" or "Substitutable Medical Apps" or "Clinical Quality Language" or "CQL" or "FHIR" or "Fast Healthcare Interoperability Resources" or "CDS Hooks" or "Clinical Decision Support Hooks")

Articles that were not returned via PubMed search but which were known to the authors to be relevant were also included in the review.

\subsection{Literature Screening}

Citation titles and abstracts were screened by two reviewers working independently (PT and CR). Next, the full text of included articles was reviewed to confirm eligibility. Conflicts were resolved via discussion to achieve consensus between the reviewers. Where uncertainty remained after discussion, articles were brought to the full team for resolution. The software Rayyan was used to manage the process (QCRI, Doha).

\subsection{Data Extraction}

Extraction fields were defined in a study protocol through discussion and consensus with the full research team. Definitions of data extraction fields can be found in the online supplement. Ten articles $(23 \%)$ were randomly selected and reviewed by the two 
reviewers simultaneously to establish consistency in the data extraction process. Data in the remaining articles were then abstracted individually by the same two reviewers. To describe these CDS evaluations, we used the "problem-intervention-comparison-outcome" (PICO) framework. Study design was categorized using a simplified version of the typology designed by Parab and Bhalerao [16]. To categorize the development of CDS technologies, we distinguished between three stages: design (architecture design and very early prototype development); piloting (creation of a functional prototype and potential evaluation); and deployment (use of the technology in its intended healthcare setting). To assess the types of CDS implemented in the selected studies, we used a simplified version of the typology offered by Wright et al. [17]. Results were summarized in a tabular format.

\section{Results}

The PubMed search yielded 290 articles. Two highly similar articles reporting on the same study were identified and the article containing less information was removed. One relevant article known to the authors but not returned via the PubMed search was added to the set. Of 290 articles screened using the title and abstract, 83 were selected for full-text review, and 44 articles met criteria for inclusion. Across both screening phases, 173 articles were excluded for not reporting on CDS-specific technologies. Of those, 45 were focused primarily or exclusively on research applications. Another 38 were excluded because they were found to be on unrelated topics; 19 viewpoints were excluded; six articles were excluded because they reported on public health applications; and four reviews were excluded. Five non-English articles were also excluded [18-22]. The overall screening process is described in Figure 1.

Among the 44 included articles, FHIR was the most frequently investigated standard $(n=43)$, followed by SMART $(n=22)$, CQL $(n=2)$, and CDS Hooks $(n=8)$. Over half $(n=23)$ of the studies reported designing, building or deploying technologies in the U.S. The remaining 21 studies included

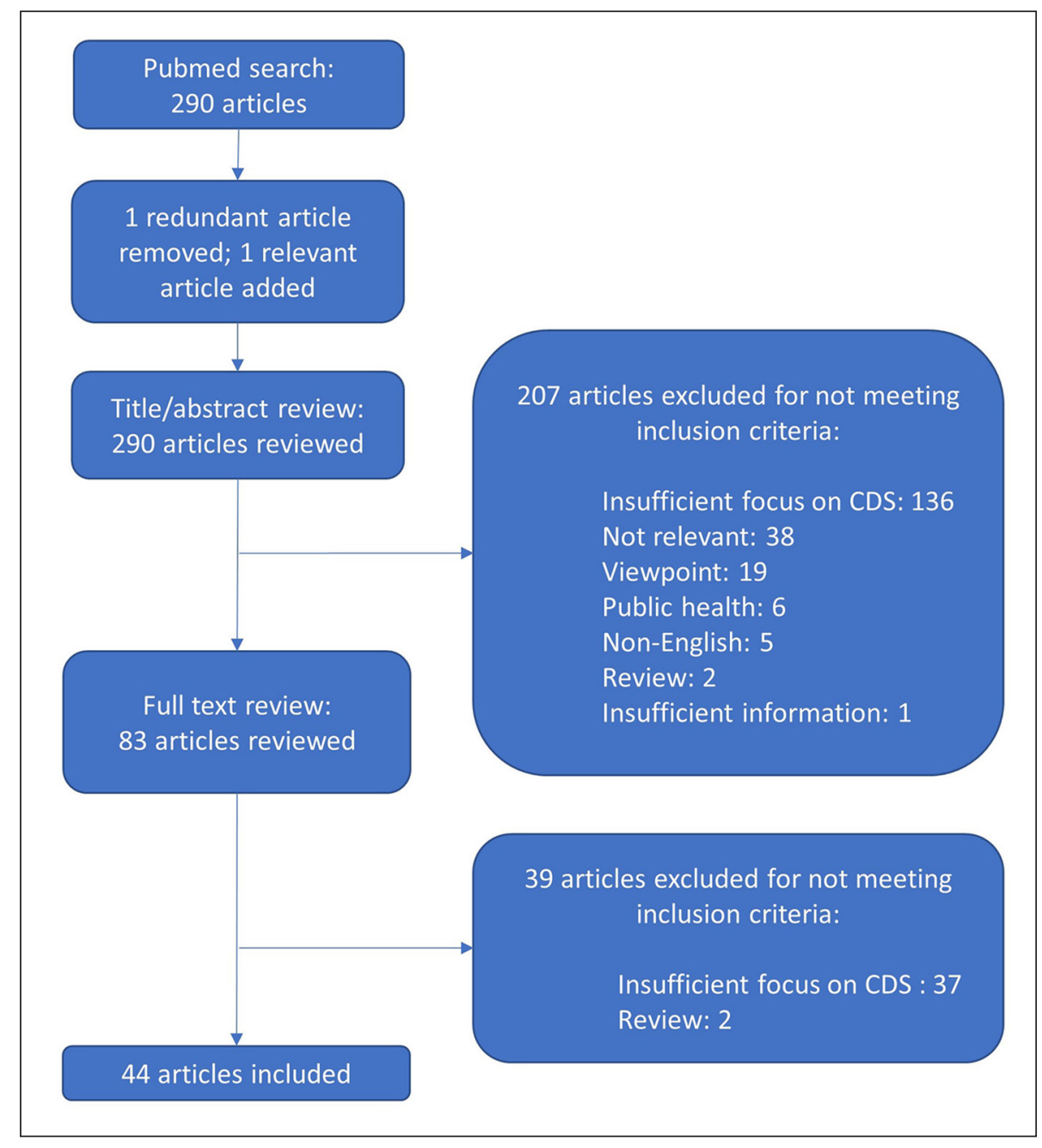

Fig. 1 Flowchart depicting article search and screening.

efforts in nine countries, including three in Russia, two each in Germany and Austria, and one each from Switzerland, Spain, Norway, Korea, Japan and Argentina. Location was not specified for six studies. Five studies reported using the Cerner ${ }^{\circledR}$ EHR, 11 reported using Epic $\AA$, and one reported using a "home-grown" EHR. No EHR was specified for 30 studies. The settings reported in the studies ranged from $18 \%$ focused solely on inpatient settings, $36 \%$ on outpatient, and $5 \%$ on both. Setting was not specified for $41 \%$ of articles. While $75 \%$ of the articles focused on healthcare professional-facing tools, $5 \%$ focused on patient-facing tools, and $16 \%$ focused on tools with components for both providers and patients. Intended users were not specified for $5 \%$ of articles. Common clinical domains included primary care $(n=9 ; 20 \%)$; oncology $(n=5 ; 11 \%)$; emergency care $(\mathrm{n}=3 ; 7 \%)$ and intensive care units $(\mathrm{n}=3 ; 7 \%)$. We found $55 \%$ of studies described CDS tools that were in the design stage, $34 \%$ in piloting stage, and $11 \%$ that have been deployed in clinical settings. Table 1 summarizes characteristics of the included articles by development stage.

An examination of temporal trends in the use of the standards of interest for CDS indicates significant growth beginning in 2018, when the number of total citations more than doubled. FHIR has been the most 


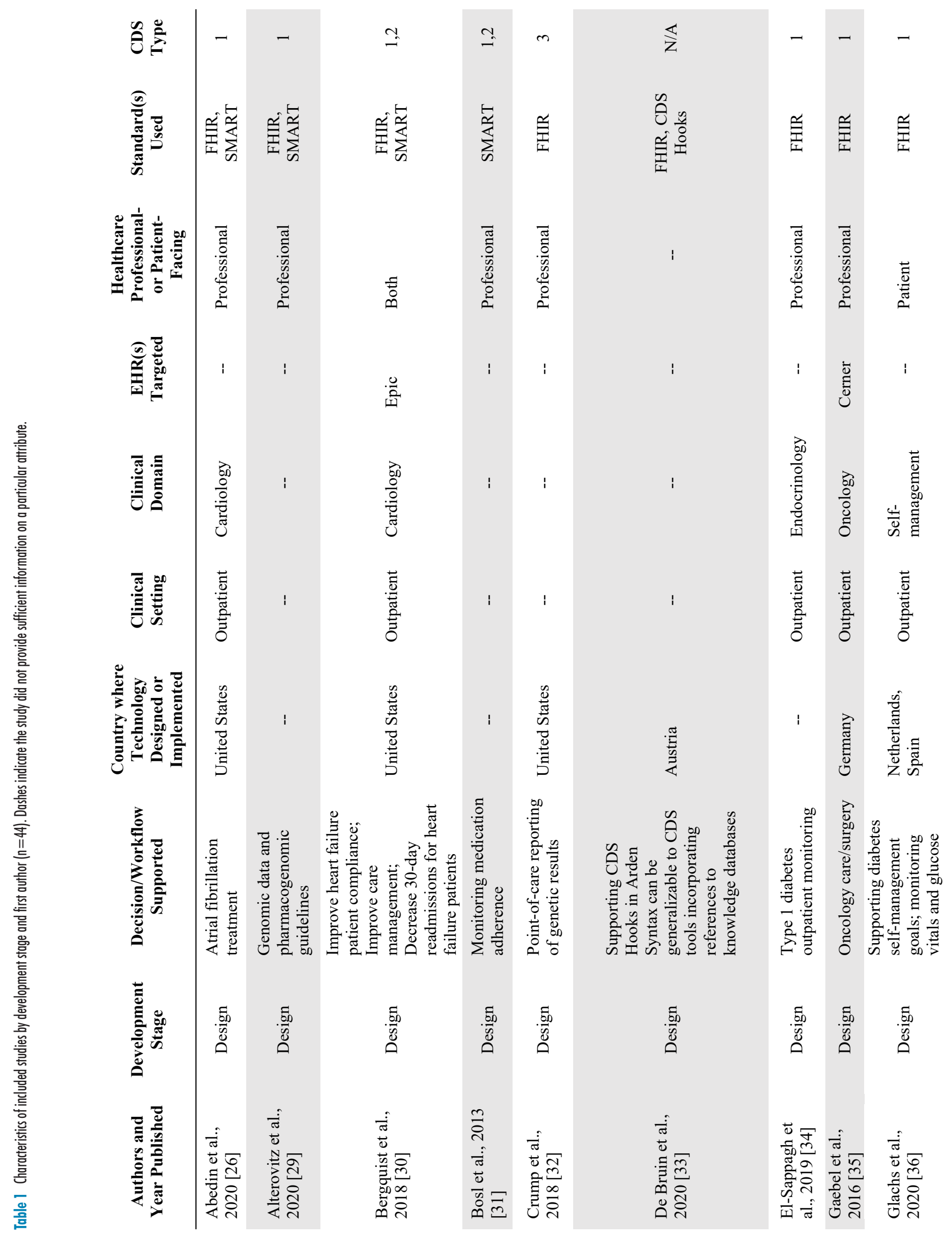




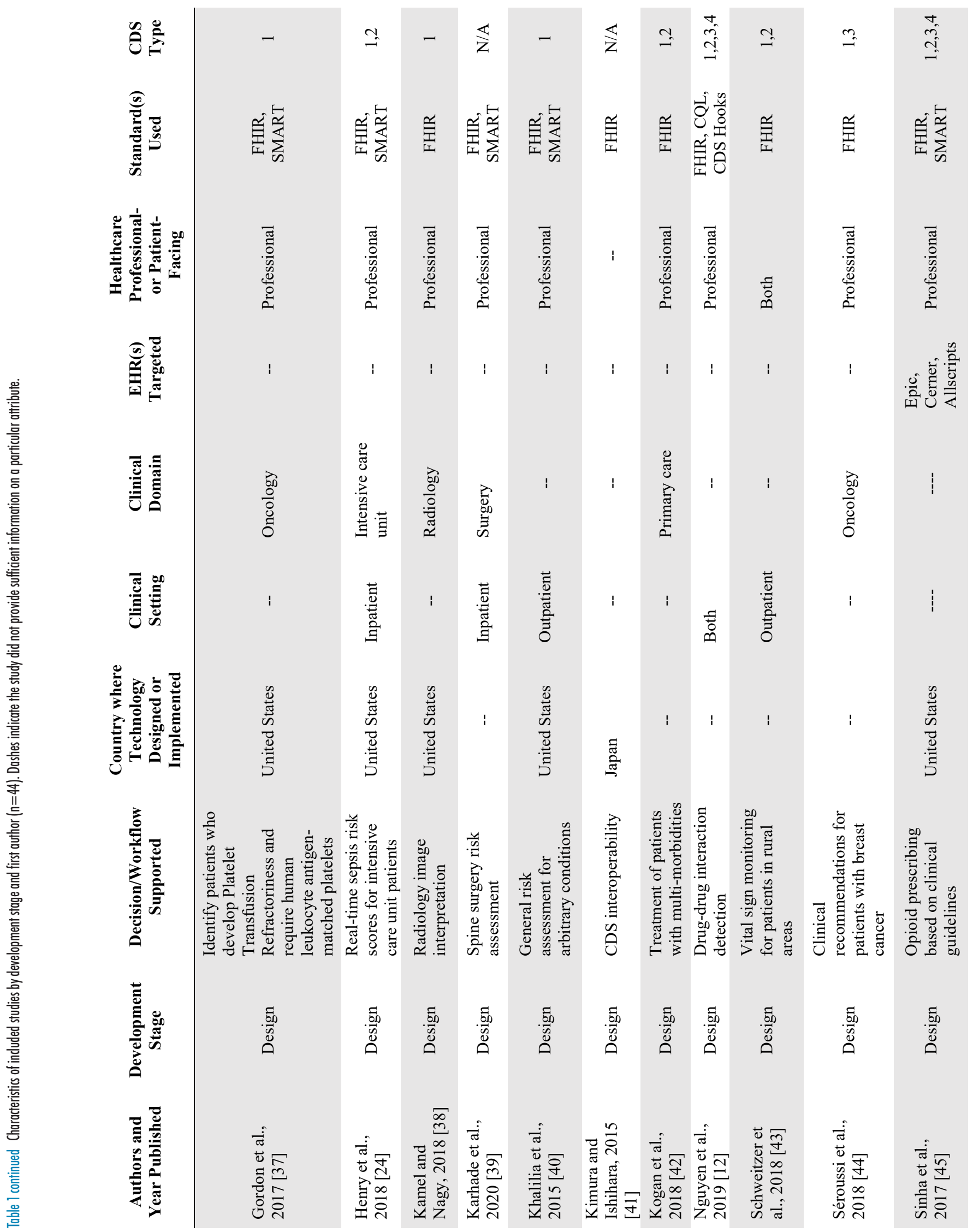




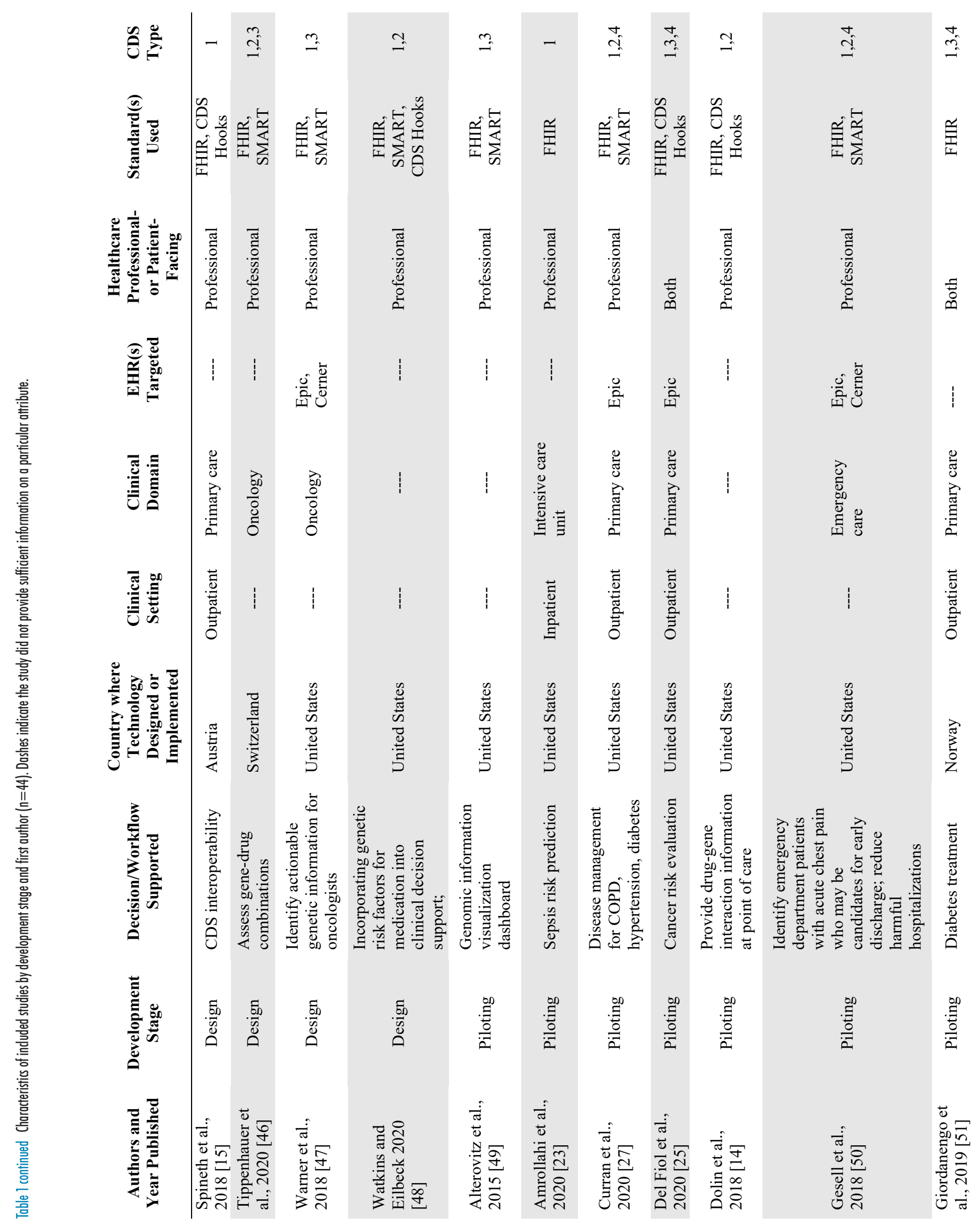




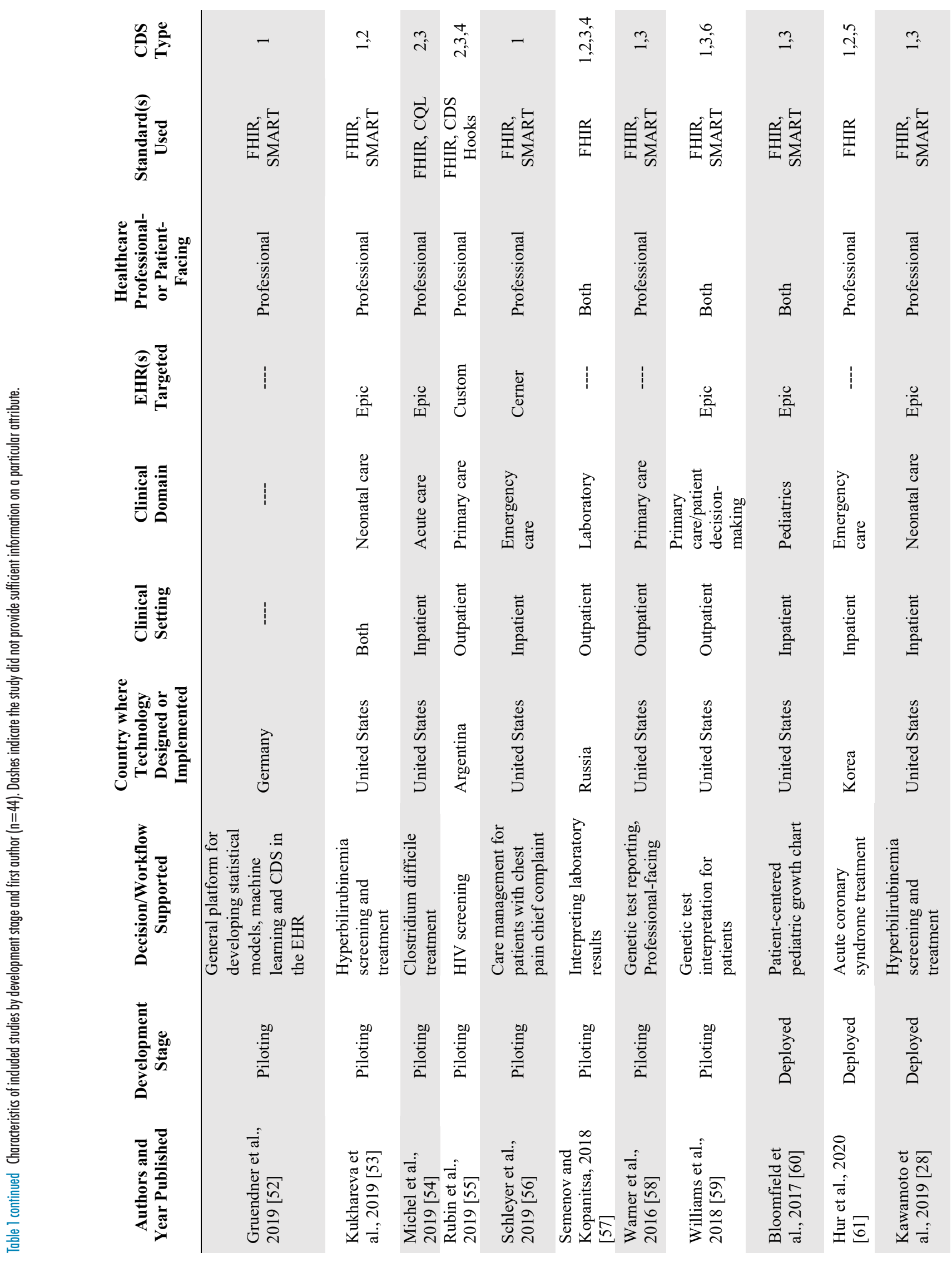


Taber et al.
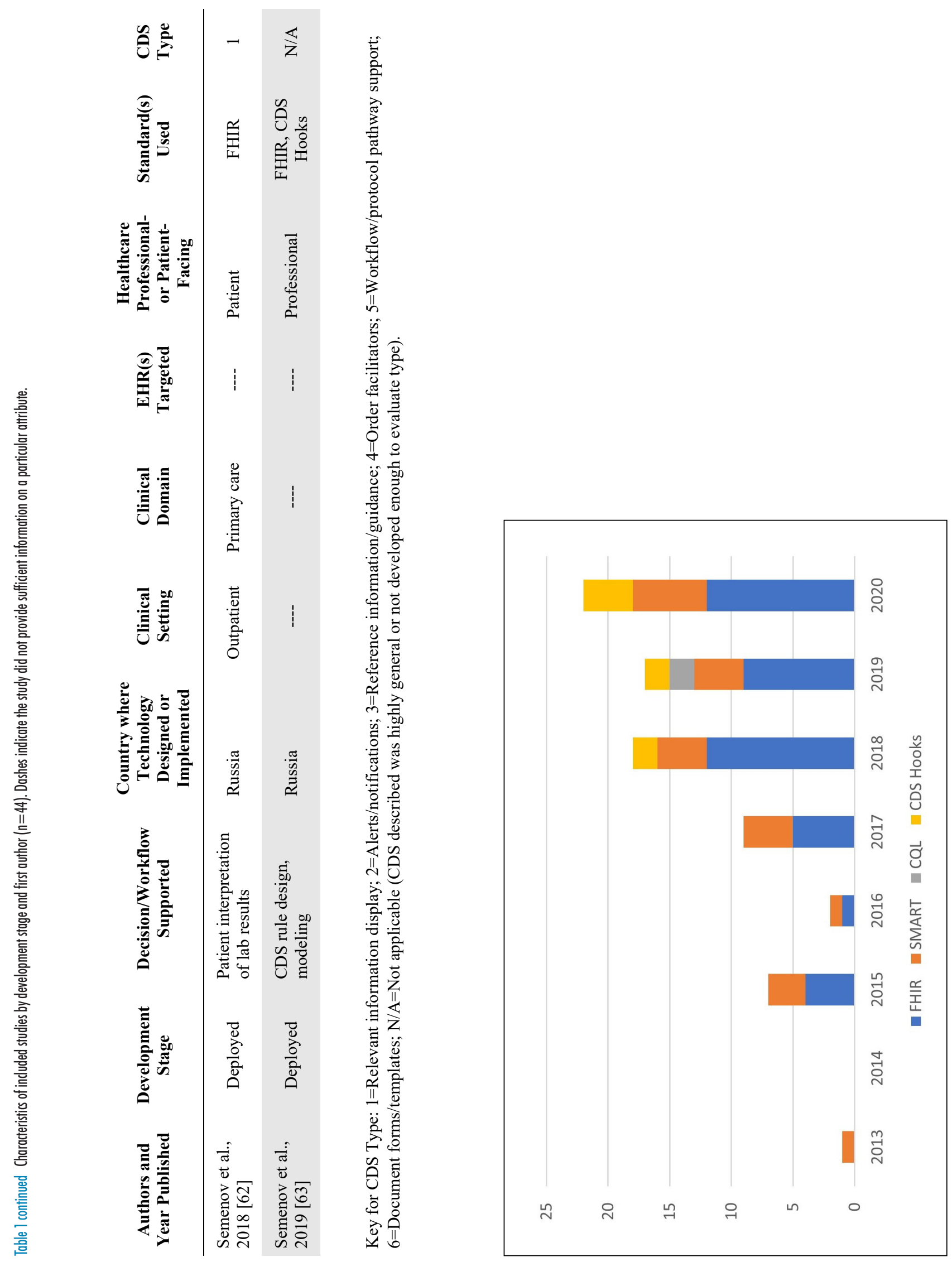
frequently studied standard by year in all years since its inception, except in 2016 , when a single SMART on FHIR study was published. Frequencies of studies investigating each standard by year since 2013 are provided in Figure 2.

Many studies involved more than one CDS type (e.g., both relevant information display and an alert). When using FHIR, SMART or CDS Hooks, relevant information display was the most common CDS type reported. Among these three standards, $33-50 \%$ also reported implementation of alerts/notifications or reference information guidance and $18-38 \%$ reported implementation of order facilitators. Workflow support and document forms or templates were rarely emphasized across any of the standards. A summary of CDS type frequencies by standard can be viewed in Table 2 .

To assess the topical foci of CDS interventions, we took a "bottom up" approach and classified topic summaries abstracted from articles into seven major groupings. "Condition management and treatment" was the most common focus of CDS using current standards and tended to focus on specific diseases $(n=15)$. "CDS infrastructure" was used to designate the development of generalizable technologies to assist in building and implementing a range of more specific CDS tools ( $\mathrm{n}=7$ ). A significant proportion of included studies focused on the development of CDS for genomics lab result interpretation $(n=7)$. Similarly, the analysis of risk factors was an important focus of work $(\mathrm{n}=6)$, including work on sepsis risk [23, 24], familial cancers [25], drug-gene interactions [14], and CHA2DS2-VASc scores [26]. Tools intended to assist clinicians or patients by monitoring particular health metrics, whether for inpatient or outpatient care, constituted a fifth category $(n=5)$. Finally, three tools for non-genomics lab result interpretation were identified, as well as one tool related to HIV screening. Table 3 summarizes the standards used by the included studies in relation to the seven topics identified, as well as the number of studies within each topic that include an evaluation.

Only $12(27 \%)$ studies reported performing any form of evaluation of their proposed technology. The most common study design

Table 2 Frequency of CDS type reported by standard.

\begin{tabular}{|l|cccc|}
\hline & FHIR ( $\mathrm{n}=43)$ & SMART ( $\mathrm{n}=22)$ & CQL $(\mathrm{n}=2)$ & CDS Hooks $(\mathrm{n}=8)$ \\
\hline CDS Type & $\mathrm{n}(\%)$ & $\mathrm{n}(\%)$ & $\mathrm{n}(\%)$ & $\mathrm{n}(\%)$ \\
Relevant Information & $35(81)$ & $20(91)$ & $1(50)$ & $5(63)$ \\
Display & $16(37)$ & $9(41)$ & $2(100)$ & $4(50)$ \\
Alerts/ Notifications & $16(37)$ & $7(32)$ & $2(100)$ & $3(38)$ \\
Reference Information/ & & $3(14)$ & $1(50)$ & $3(38)$ \\
Guidance & $8(19)$ & $0(0)$ & $0(0)$ & $0(0)$ \\
Order facilitators & $1(2)$ & $1(5)$ & $0(0)$ & $0(0)$ \\
Workflow/Protocol & $1(2)$ & & & \\
Pathway Support & & & & \\
Documentation Forms/ & & & & \\
Templates & & &
\end{tabular}

Table 3 Summary of topics addressed by standards used and evaluation performed.

\begin{tabular}{|c|c|c|c|c|c|}
\hline \multirow{3}{*}{ Topic } & \multicolumn{3}{|c|}{ Standards } & \multirow[b]{2}{*}{ CDS Hooks } & \multirow{3}{*}{$\begin{array}{c}\text { \# Studies Reporting } \\
\text { Evaluations } \\
n(\%)\end{array}$} \\
\hline & FHIR & SMART & CQL & & \\
\hline & n (\%) & n (\%) & $n(\%)$ & $\mathrm{n}(\%)$ & \\
\hline $\begin{array}{l}\text { Condition Management and Treatment }(n=15) \\
{[12,27,28,30,31,35,36,42,44,45,50,} \\
53,54,56,58]\end{array}$ & $14(93)$ & $9(60)$ & 2 (13) & $1(7)$ & $4(27)$ \\
\hline $\begin{array}{l}\text { CDS Infrastructure }(n=7) \\
{[15,33,40,41,52,60,63]}\end{array}$ & $7(100)$ & $3(43)$ & $0(0)$ & $3(43)$ & $1(14)$ \\
\hline $\begin{array}{l}\text { Lab Result Interpretation -- Genomics }(n=7) \\
{[29,32,46,48,49,58,59]}\end{array}$ & $7(100)$ & $6(86)$ & $0(0)$ & $1(14)$ & 1 (14) \\
\hline $\begin{array}{l}\text { Risk Factor Analysis }(n=6) \\
{[14,23-26,39]}\end{array}$ & $6(100)$ & $3(50)$ & $0(0)$ & $2(33)$ & $1(17)$ \\
\hline $\begin{array}{l}\text { Health Metric Monitoring }(n=5) \\
{[34,37,43,51,61]}\end{array}$ & $5(100)$ & $1(20)$ & $0(0)$ & $0(0)$ & $2(40)$ \\
\hline $\begin{array}{l}\text { Lab Result Interpretation -- Other }(n=3) \\
{[38,57,62]}\end{array}$ & $3(100)$ & $0(0)$ & $0(0)$ & $0(0)$ & $2(67)$ \\
\hline $\begin{array}{l}\text { HIV Screening }(n=1) \\
\text { [55] }\end{array}$ & $1(100)$ & $0(0)$ & $0(0)$ & $1(100)$ & $1(100)$ \\
\hline
\end{tabular}

was observational $(n=8 ; 67 \%)$, with three studies $(25 \%)$ reporting CDS needs evaluations. We identified only two studies with randomized components reporting on the use of any of the standards of interest (Curran et al. [27] and Kawamoto et al. [28]). Two
(17\%) articles reporting evaluations were in the design stage, seven (58\%) were in the piloting stage, and a further three $(25 \%)$ were deployed in real clinical settings. Table 4 summarizes the characteristics of studies that reported an evaluation. 


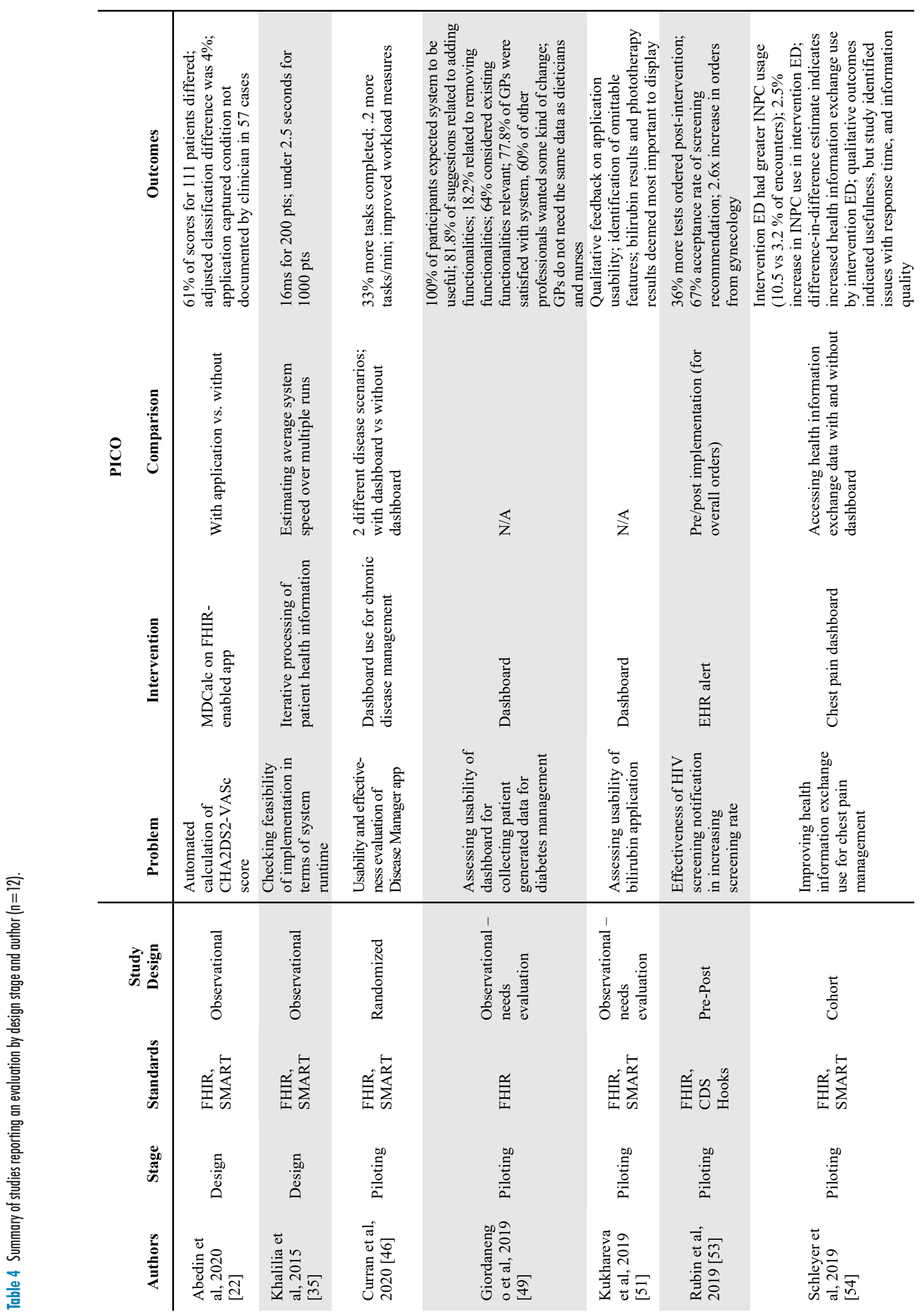




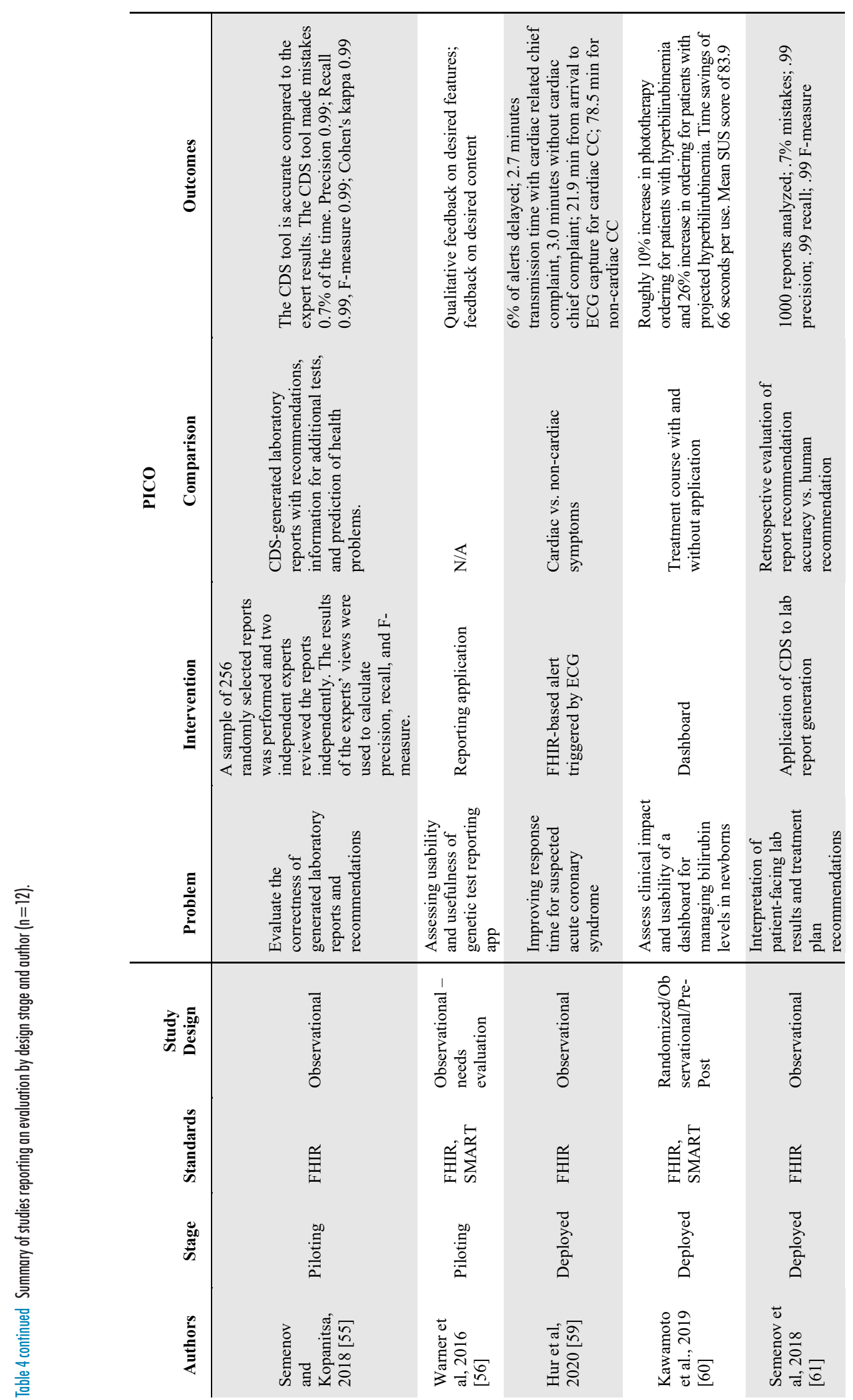




\section{Discussion}

The large proportion of studies in the design or pilot stages (55\% and 34\%, respectively) highlights the newness of the technology and ongoing efforts to implement the key CDS standards. Similarly, the low proportion of studies reporting any form of evaluation (27\%), and the fact that only two studies with randomized evaluation components exist, highlight the continued need for building a strong evidence base around the performance of these standards in practice.

While work with these standards is clearly still in its early stages, there are reasons to be optimistic about their development. Since 2018, we found the use of new healthcare interoperability standards in CDS to be a highly active area of research. Predictably, tools for condition management and treatment are the most common uses for the standards we examined. However, the development of new infrastructures to support a wide range of platform-agnostic CDS development, as well as support for the highly technical domain of genomics lab interpretation, show the promise of these new standards. The high proportion of studies making some use of FHIR in and outside the United States suggests general buy-in to the standard. The low number of studies focused on CDS Hooks and CQL suggests that it is still too early in their life cycles to evaluate their uptake, though we note that CQL's adoption by CMS may encourage widespread implementation in the future.

This survey had some limitations. Although our article screening was modeled on, and generally compliant with, scoping review methodology, the search strategy, data extraction, and data analysis were more informal. For example, we searched only one database, our search strategy was not developed with assistance from an expert librarian, and the data analysis did not follow standard scoping review methods. As another limitation, the study was conducted over a short period in which we observed rapid development of the literature; the most recent article captured in the search was published in August, 2020 [27]. It is highly likely that new studies have been published that were not captured in our October 21, 2020 literature search at the time of writing. Nevertheless, we believe that this review provides a useful representation of the current state-ofthe-art related to the implementation of new CDS standards, and a point of reference for future assessments of the field.

\section{Conclusion}

The diversity of topics and approaches identified as well as rapid growth in the recent literature highlights the potential utility of and increased interest in the adoption of this emerging set of standards for CDS. The infrequency of reported evaluations, as well as the high number of studies in the design or piloting stage, indicate that use of these technologies for CDS is still nascent and requires further study. Informaticists will require a stronger evidence base regarding the use of these standards in CDS design and implementation to make design decisions going forward.

\section{References}

1. Osheroff JA, Teich J, Levick D, Saldana L, Velasco F, Sittig D, et al. Improving outcomes with clinical decision support: an implementer's guide. HIMSS Publishing; 2012.

2. Greenes RA, Bates DW, Kawamoto K, Middleton B, Osheroff J, Shahar Y. Clinical decision support models and frameworks: Seeking to address research issues underlying implementation successes and failures. J Biomed Inform 2018;78:134-43.

3. Blumenthal D, Tavenner M. The "meaningful use" regulation for electronic health records. N Engl J Med 2010;363(6):501-4.

4. Braunstein ML. Health Care in the Age of Interoperability: The Potential and Challenges. IEEE Pulse 2018;9(5):34-6.

5. Braunstein ML. Healthcare in the Age of Interoperability: The Promise of Fast Healthcare Interoperability Resources. IEEE Pulse 2018;9(6):24-7.

6. Braunstein ML. Health Care in the Age of Interoperability Part 6: The Future of FHIR. IEEE Pulse 2019;10(4):25-7.

7. Strasberg HR, Rhodes B, Del Fiol G, Jenders RA, Kawamoto K. Contemporary clinical decision support standards using Health Level Seven International (HL7) Fast Healthcare Interoperability Resources (FHIR). J Am Med Inform Assoc 2021 Jun 8;ocab070.

8. Lehne M, Luijten S, Vom Felde Genannt Imbusch P, Thun S. The Use of FHIR in Digital Health - A Review of the Scientific Literature. Stud Health Technol Inform 2019;267:52-8.

9. Mandel JC, Kreda DA, Mandl KD, Kohane IS, Ramoni RB. SMART on FHIR: a standards-based, interoperable apps platform for electronic health records. J Am Med Inform
Assoc 2016;23(5):899-908.

10. Mandl KD, Mandel JC, Murphy SN, Bernstam VE, Ramoni RL, Kreda DA, et al. The SMART Platform: early experience enabling substitutable applications for electronic health records. J Am Med Inform Assoc 2012;19(4):597-603.

11. Li M, Zhi Y, Lu X, Cai H. Extending CQL with openEHR to Express Clinical Quality Indicators. Stud Health Technol Inform 2019;264:1853-4.

12. Nguyen BP, Reese T, Decker S, Malone D, Boyce RD, Beyan O. Implementation of Clinical Decision Support Services to Detect Potential Drug-Drug Interaction Using Clinical Quality Language. Stud Health Technol Inform 2019;264:724-8.

13. HL7. Clinical Quality Language (CQL) [Internt]. 2019. [cited 2021 May 14]. Available from: https:// cql.hl7.org/

14. Dolin RH, Boxwala A, Shalaby J. A Pharmacogenomics Clinical Decision Support Service Based on FHIR and CDS Hooks. Methods Inf Med 2018;57(S 02):e115-e123.

15. Spineth M, Rappelsberger A, Adlassnig KP. Implementing CDS Hooks Communication in an Arden-Syntax-Based Clinical Decision Support Platform. Stud Health Technol Inform 2018;255:165-9.

16. Parab S, Bhalerao S. Study designs. Int J Ayurveda Res 2010;1(2):128-31.

17. WrightA, Sittig DF, Ash JS, Feblowith J, Meltzer S, McMullen C, et al. Development and evaluation of a comprehensive clinical decision support taxonomy: comparison of front-end tools in commercial and internally developed electronic health record systems. JAm Med Inform Assoc 2011;18(3):23242.

18. Bauer J, Rohner-Rojas S, Holderried M. [Consent management and workflows for cross-sectoral patient records and teleconsultations]. Radiologe 2020;60(5):430-9. German.

19. Bauer J, Rohner-Rojas S, Holderried M. [Cross-enterprise interoperability : Challenges and principles for technical implementation]. Radiologe. 2020;60(4):334-341. German.

20. Chen Y, Lu Y, Wang YN, Lin Z-C, Gu W, Tan L, et al. [Effect of compound qingqin liquid on the expression of toll-like receptor in the renal tissue of rats with urate nephropathy]. Zhongguo Zhong Xi Yi Jie He Za Zhi 2014;34(6):722-7. Chinese.

21. Djian J, Lellouch AG, Botter C, Levy J, Burgun A, Hivelin M, et al. [Clinical photography by smartphone in plastic surgery and protection of personal data: Development of a secured platform and application on 979 patients]. Ann Chir Plast Esthet 2019;64(1):33-43. French.

22. Shang XZ, Ma WG, Chen Y,Lu Y, Wang Y-N, Xu $\mathrm{Y}-\mathrm{M}$, et al. [Effect of compound qingqin liquid on the expression levels of ang II and COX-2 mRNA transcription and protein expression in the renal tissue of uric acid nephropathy rats: an experimental study]. Zhongguo Zhong Xi Yi Jie He Za Zhi 2014;34(7):819-25. Chinese.

23. Amrollahi F, Shashikumar SP, Kathiravelu P, Sharma A, Nemati S. AIDEx - An Open-source Platform for Real-Time Forecasting Sepsis and A Case Study on Taking ML Algorithms to Production. Annu Int Conf IEEE Eng Med Biol Soc 2020;2020:5610-4.

24. Henry JR, Lynch D, Mals J, Shashikumar SP, Hold- 
er A, Sharma A, et al. A FHIR-Enabled Streaming Sepsis Prediction System for ICUs. Annu Int Conf IEEE Eng Med Biol Soc 2018;2018:4093-6.

25. Del Fiol G, Kohlmann W, Bradshaw RL, Weir CR, Flynn M, Hess R, et al. Standards-Based Clinical Decision Support Platform to Manage Patients Who Meet Guideline-Based Criteria for Genetic Evaluation of Familial Cancer. JCO Clin Cancer Inform 2020;4:1-9.

26. Abedin Z, Hoerner R, Habboushe J, Lu Y, Kawamoto $\mathrm{K}$, Warner PB, et al. Implementation of a Fast Healthcare Interoperability Resources-Based Clinical Decision Support Tool for Calculating CHA(2)DS(2)-VASc Scores. Circ Cardiovasc Qual Outcomes 2020;13(2):e006286.

27. Curran RL, Kukhareva PV, Taft T, Weir CR, Reese TJ, Nanjo C, et al. Integrated displays to improve chronic disease management in ambulatory care: A SMART on FHIR application informed by mixed-methods user testing. J Am Med Inform Assoc 2020;27(8):1225-34.

28. Kawamoto K, Kukhareva P, Shakib JH, Kramer H, Rodriguez S, Warnter PB, Shields D, et al. Association of an Electronic Health Record Addon App for Neonatal Bilirubin Management With Physician Efficiency and Care Quality. JAMA Netw Open 2019;2(11):e1915343.

29. Alterovitz G, Heale B, Jones J, Kreda D, Lin F, Liu L, et al. FHIR Genomics: enabling standardization for precision medicine use cases. NPJ Genom Med 2020;5:13

30. Bergquist T, Buie RW, Li K, Brandt P. Heart on FHIR: Integrating Patient Generated Data into Clinical Care to Reduce 30 Day Heart Failure Readmissions (Extended Abstract). AMIA Annu Symp Proc 2017;2017:2269-73.

31. Bosl W, Mandel J, Jonikas M, Ramoni RB, Kohane IS, Mandl KD. Scalable decision support at the point of care: a substitutable electronic health record app for monitoring medication adherence. Interact J Med Res 2013;2(2):e13.

32. Crump JK, Del Fiol G, Williams MS, Freimuth RR. Prototype of a Standards-Based EHR and Genetic Test Reporting Tool Coupled with HL7-Compliant Infobuttons. AMIA Jt Summits Transl Sci Proc 2018;2017:330-9.

33. de Bruin JS, Rappelsberger A, Adlassnig KP, Gawrylkowicz J. Exploring Methods of Implementing Arden Syntax for CDS Hooks. Stud Health Technol Inform 2020;271:191-8.

34. El-Sappagh S, Ali F, Hendawi A, Jang JH, Kwak KS. A mobile health monitoring-and-treatment system based on integration of the SSN sensor ontology and the HL7 FHIR standard. BMC Med Inform Decis Mak 2019;19(1):97.

35. Gaebel J, Cypko MA, Lemke HU. Accessing Patient Information for Probabilistic Patient Models Using Existing Standards. Stud Health Technol Inform 2016;223:107-112.

36. Glachs D, Namli T, Jung O, Strohmeier F, Ploessnig M, Rodriguez G. FHIR Driven Self-Management Support System for Diabetes. Stud Health Technol Inform 2020;270:1291-2.

37. Gordon WJ, Baronas J, Lane WJ. A FHIR Human Leukocyte Antigen (HLA) Interface for Platelet Transfusion Support. Appl Clin Inform 2017:8(2):603-11

38. Kamel PI, Nagy PG. Patient-Centered Radiology with FHIR: an Introduction to the Use of FHIR to Offer Radiology a Clinically Integrated Platform. J Digit Imaging 2018;31(3):327-33.

39. Karhade AV, Schwab JH, Del Fiol G, Kawamoto $\mathrm{K}$. SMART on FHIR in spine: integrating clinical prediction models into electronic health records for precision medicine at the point of care. Spine J 2020 Jun 26;S1529-9430(20)30820-2.

40. Khalilia M, Choi M, Henderson A, Iyengar S, Braunstein M, Sun J. Clinical Predictive Modeling Development and Deployment through FHIR Web Services. AMIA Annu Symp Proc 2015;2015:717-26.

41. Kimura E, Ishihara K. Internal domain-specific language based on Arden Syntax and FHIR. Stud Health Technol Inform 2015;216:955.

42. Kogan A, Tu SW, Peleg M. Goal-driven management of interacting clinical guidelines for multimorbidity patients. AMIA Annu Symp Proc 2018;2018:690-9.

43. Schweitzer M, Huber L, Gorfer T, Hörbst A. An Approach for Dynamic Vital Parameter Monitoring - Prototype Development. Stud Health Technol Inform 2018;251:47-50.

44. Séroussi B, Guézennec G, Lamy JB, et al. Reconciliation of multiple guidelines for decision support: a case study on the multidisciplinary management of breast cancer within the DESIREE project. AMIA Annu Symp Proc 2017;2017:1527-36.

45. Sinha S, Jensen M, Mullin S, Elkin PL. Safe Opioid Prescription: A SMART on FHIR Approach to Clinical Decision Support. Online J Public Health Inform 2017;9(2):e193.

46. Tippenhauer K, Philips M, Largiadèr CR, Sariyar M, Bürkle T. Integrating Pharmacogenetic Decision Support into a Clinical Information System. Stud Health Technol Inform 2020;270:618-22.

47. Warner JL, Prasad I, Bennett M, Arniella M, Beeghly-Fadiel A, Mandl KD, et al. SMART Cancer Navigator: A Framework for Implementing ASCO Workshop Recommendations to Enable Precision Cancer Medicine. JCO Precis Oncol 2018;2018:PO.17.00292.

48. Watkins M, Eilbeck K. FHIR Lab Reports: using SMART on FHIR and CDS Hooks to increase the clinical utility of pharmacogenomic laboratory test results. AMIA Jt Summits Transl Sci Proc 2020;2020:683-92.

49. Alterovitz G, Warner J, Zhang P, Chen Y, Ullman-Cullere M, Kreda D, et al. SMART on FHIR Genomics: facilitating standardized clinico-genomic apps. J Am Med Inform Assoc 2015;22(6):1173-8

50. Gesell SB, Golden SL, Limkakeng AT Jr, Carr CM, Matuskowitz, Smith LM, et al. Implementation of the HEART Pathway: Using the Consolidated Framework for Implementation Research. Crit Pathw Cardiol 2018;17(4):191-200.

51. Giordanengo A, Årsand E, Woldaregay AZ, Bradway M, Gottland A, Hartvigsen G, et al. Design and Prestudy Assessment of a Dashboard for Presenting Self-Collected Health Data of Patients With Diabetes to Clinicians: Iterative Approach and Qualitative Case Study. JMIR Diabetes 2019;4(3):e14002.

52. Gruendner J, Schwachhofer T, Sippl P, Wolf N, Erpenbeck M, Gulden C, et al. KETOS: Clinical decision support and machine learning as a service
- A training and deployment platform based on Docker, OMOP-CDM, and FHIR Web Services. PLoS One 2019;14(10):e0223010.

53. Kukhareva P, Warner P, Rodriguez S, Kramer H, Weir C, Nanjo C, et al. Balancing Functionality versus Portability for SMART on FHIR Applications: Case Study for a Neonatal Bilirubin Management Application. AMIA Annu Symp Proc 2019;2019:562-71.

54. Michel J, Flores E, Mull N, Tsou AY. AHRQ Methods for Effective Health Care. In: Translation of a C. difficile Treatment Clinical Pathway Into Machine-Readable Clinical Decision Support Artifacts Prototyped for Electronic Health Record Integration. Rockville (MD). Agency for Healthcare Research and Quality (US); 2019.

55. Rubin L, López NP, Gaiera A, Campos F, Luna D, de Quirós FBG. Development, Implementation and Preliminary Results of an Electronic Reminder for HIV Screening Using a Service Oriented Architecture. Stud Health Technol Inform 2019;264:763-7.

56. Schleyer TKL, Rahurkar S, Baublet AM, Kochmann M, Ning X, Martin DK, et al. Preliminary evaluation of the Chest Pain Dashboard, a FHIRbased approach for integrating health information exchange information directly into the clinical workflow. AMIA Jt Summits Transl Sci Proc 2019;2019:656-64.

57. Semenov I, Kopanitsa G. Decision Support System Based on FHIR Profiles. Stud Health Technol Inform 2018;249:117-21.

58. Warner JL, Rioth MJ, Mandl KD, Mandel JC, Kreda DA, Kohane IS, et al. SMART precision cancer medicine: a FHIR-based app to provide genomic information at the point of care. J Am Med Inform Assoc 2016;23(4):701-10.

59. Williams MS, Kern MS, Lerch VR, Billet J, Williams JL, Moore GJ. Implementation of a patient-facing genomic test report in the electronic health record using a web-application interface. BMC Med Inform Decis Mak 2018;18(1):32.

60. Bloomfield RA Jr, Polo-Wood F, Mandel JC, Mandl KD. Opening the Duke electronic health record to apps: Implementing SMART on FHIR. Int J Med Inform 2017;99:1-10.

61. Hur S, Lee J, Kim T, Choi JS, Kang M, Chang DK, et al. An Automated Fast Healthcare Interoperability Resources-Based 12-Lead Electrocardiogram Mobile Alert System for Suspected Acute Coronary Syndrome. Yonsei Med J 2020;61(5):416-22.

62. Semenov I, Kopanitsa G, Denisov D, Alexandr Y, Osenev R, Andreychuk Y. Patients Decision Aid System Based on FHIR Profiles. J Med Syst 2018;42(9): 166.

63. Semenov I, Osenev R, Gerasimov S, Kopanitsa G, Denisov D, Andreychuk Y. Experience in Developing an FHIR Medical Data Management Platform to Provide Clinical Decision Support. Int J Environ Res Public Health 2019;17(1):73.

\section{Correspondence to:}

Peter Taber, PhD

Department of Biomedical Informatics

University of Utah

421 Wakara Way

Salt Lake City, UT 84112, USA

E-mail: peter.taber@hsc.utah.edu 